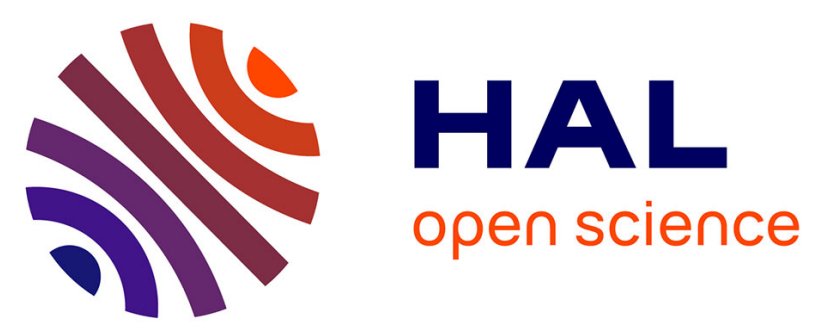

\title{
Conformational landscape and inertial defect of methoxyphenol isomers studied by mm-wave spectroscopy and quantum chemistry calculations
}

Atef Jabri, Cédric Bray, Gaël Mouret, Robin Bocquet, A. Cuisset, Guillaume Dhont, Francis Hindle, Anthony Roucou, Daniele Fontanari

\section{To cite this version:}

Atef Jabri, Cédric Bray, Gaël Mouret, Robin Bocquet, A. Cuisset, et al.. Conformational landscape and inertial defect of methoxyphenol isomers studied by mm-wave spectroscopy and quantum chemistry calculations. Journal of Chemical Physics, 2019, 150, pp.104303. 10.1063/1.5089426 . hal-03173780

\author{
HAL Id: hal-03173780 \\ https://hal.science/hal-03173780
}

Submitted on 18 Mar 2021

HAL is a multi-disciplinary open access archive for the deposit and dissemination of scientific research documents, whether they are published or not. The documents may come from teaching and research institutions in France or abroad, or from public or private research centers.
L'archive ouverte pluridisciplinaire HAL, est destinée au dépôt et à la diffusion de documents scientifiques de niveau recherche, publiés ou non, émanant des établissements d'enseignement et de recherche français ou étrangers, des laboratoires publics ou privés. 


\section{Conformational landscape and inertial defect of methoxyphenol isomers studied by $\mathrm{mm}$-wave spectroscopy and quantum chemistry calculations}

Cite as: J. Chem. Phys. 150, 104303 (2019); https://doi.org/10.1063/1.5089426

Submitted: 18 January 2019 . Accepted: 21 February 2019 . Published Online: 13 March 2019

A. Jabri, D. Fontanari (D), A. Roucou (D), C. Bray, F. Hindle (D), G. Dhont, G. Mouret (D), R. Bocquet, and A. Cuisset (iD)
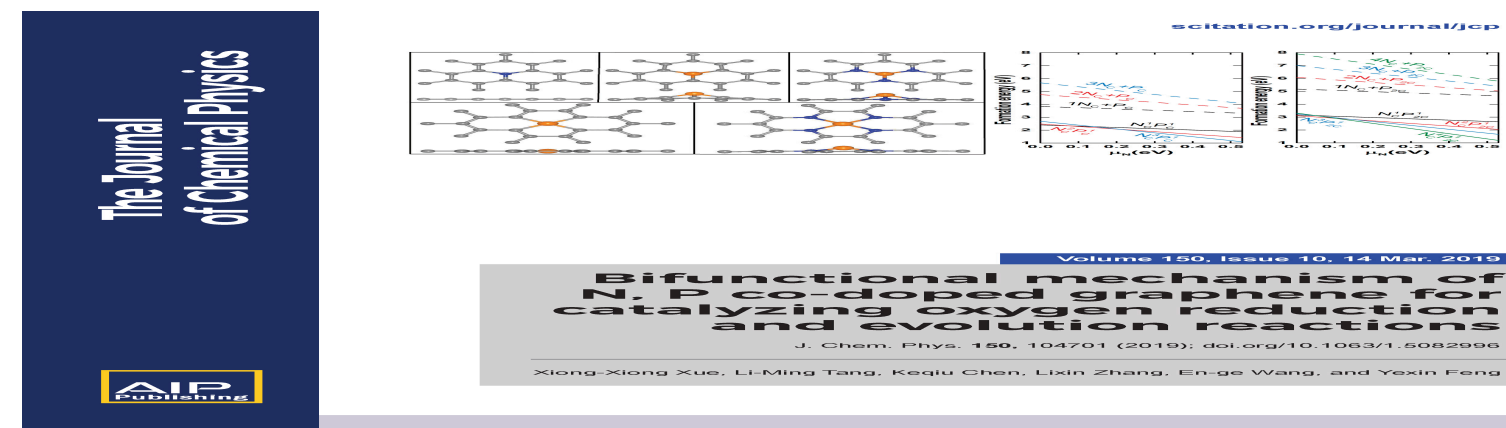


\title{
Conformational landscape and inertial defect of methoxyphenol isomers studied by $\mathrm{mm}$-wave spectroscopy and quantum chemistry calculations
}

\author{
Cite as: J. Chem. Phys. 150, 104303 (2019); doi: 10.1063/1.5089426 \\ Submitted: 18 January 2019 Accepted: 21 February 2019 • \\ Published Online: 13 March 2019
}

\begin{abstract}
A. Jabri,' D. Fontanari,
A. Roucou,

C. Bray, ${ }^{1,2}$ F. Hindle,

G. Dhont, ${ }^{1}$ G. Mouret,

R. Bocquet,
\end{abstract} and A. Cuisset ${ }^{1, a)}$ (D)

\begin{abstract}
AFFILIATIONS
${ }^{1}$ Laboratoire de Physico-Chimie de l'Atmosphère, CNRS EA-4493, Université du Littoral Côte d'Opale, 59140 Dunkerque, France

${ }^{2}$ Université de Bordeaux, Institut des Sciences Moléculaires, F-33400 Talence, France
\end{abstract}

a) Electronic mail: arnaud.cuisset@univ-littoral.fr. Tel: +33 328237613

\begin{abstract}
Because methoxyphenols (MP) are emitted in significant quantities during biomass fires and contribute to the secondary organic aerosols formation which impacts the climate, their gas phase monitoring in the atmosphere is crucial and requires accurate rovibrational cross sections determined with a good knowledge of their ground state (GS) and vibrationally excited state (ES) molecular parameters. Therefore, the rotational spectra of the two isomers, 2-MP (guaiacol) and 4-MP (mequinol), have been measured in absorption and in emission at room temperature using a frequency multiplication chain and a mm-wave Fourier transform chirped-pulse spectrometer, respectively. Guided by quantum chemistry calculations, the conformational landscape has been characterised and the observation of only one rotamer in the spectra of 2-MP and 4-MP has been explained. For 2-MP, the most stable conformation is justified by an intramolecular $\mathrm{O}-\mathrm{H} \cdots \mathrm{OCH}_{3}$ hydrogen-bond which has been characterised by a topology analysis of the electron density. In a global fit including more than 30000 line assignments, rotational and quartic centrifugal constants of the GS and the three lowest energy ES have been determined allowing to reproduce the millimeter-wave spectra at the experimental accuracy. The same work has been performed on the cis-rotamer of 4-MP highlighting some perturbations marring the fit quality for two vibrationally ES. Finally, the isomeric dependence of the negative inertial defect $\Delta I$ agrees with that of the lowest energy out of plane mode $v_{45}$, and the variation of $\Delta I$ with the degree of vibrational excitation allows a fine estimation of $\mathrm{v}_{45}=1$ vibrational wavenumber.
\end{abstract}

Published under license by AIP Publishing. https://doi.org/10.1063/1.5089426

\section{INTRODUCTION}

Methoxyphenols are oxygenated aromatic compounds present as building blocks in natural lignin which play a key role in the cellular formation of wood and barks. These biogenic volatile organic compounds (BVOCs) are released in significant quantities during biomass fires (including natural fires and residential wood heating). 2-methoxyphenol (2-MP) and 2,6-dimethoxyphenol commonly named guaiacol and syringol, respectively, are the two main products of lignin pyrolysis with emission rates varying from $0.9 \mathrm{~g}$ to $4.2 \mathrm{~g}$ per $\mathrm{kg}$ of burned vegetation. ${ }^{7}$ They are semi-volatile products principally detected in the gas phase and are able to chemically evolve in the atmosphere via degradation processes. Several studies have been performed in order to determine the kinetics and the reactivity intermediates involved in the tropospheric oxidation processes and the subsequent formation of secondary organic aerosols (SOA) which impact the climate. ${ }^{2}$ It is well established that aromatic oxygenated compounds such as methoxyphenols have a strong potential for the formation of SOA with specific hygroscopic properties depending on the ability to form intermolecular hydrogen bonds. ${ }^{3}$

Indeed, depending on the isomer and the conformer forms, methoxyphenols, as other BVOCs, may present intramolecular hydrogen bond preventing or not the formation of intermolecular hydrogen bonds (HB) with water or other molecular environments. ${ }^{4}$ 
In 2-MP, the hydroxyl and methoxy groups in "ortho" position may form an intramolecular HB; whereas, in 3-methoxyphenol (3-MP) and 4-methoxyphenol (4-MP, commonly called mequinol), they are too distant to interact directly through space, which enhances the conformational flexibility and the possibility to observe several stable rotamers. Therefore, the conformational landscape of MP has been the subject of several experimental and theoretical studies ${ }^{4-9}$ showing a clear dependence on the isomeric forms.

Rotationally resolved electronic spectroscopy in molecular beam has provided the most complete conformational analyses on the MP isomers. Unlike 2-MP where UV spectra were assigned to a single conformation stabilised by the formation of an intramolecular $\mathrm{HB}$ between the adjacent hydroxyl and methoxy functional groups, several stable conformers depending on the $-\mathrm{OH}$ and $-\mathrm{OCH}_{3}$ relative orientations were identified for 3-MP and 4-MP. ${ }^{6,8}$ Two iso-energetic cis and trans stable rotamers may be individually identified in 4-MP spectra due to the significant differences of the rotational constants in the ground and electronically excited states. For 3-MP, only 3 of the 4 predicted rotamers were observed and the non-observation of the fourth conformation was explained by the non-planar structure of 3-MP in the electronic singlet state by weakening the electronic transition with an unfavorable Franck-Condon factor.

Recently, our group has demonstrated that the conformational landscape of 3-MP may be fully described at room temperature in the electronic ground state (GS) using mm-wave rotational spectroscopy supported by quantum-chemical calculations. ${ }^{9}$ Compared to the previous UV studies, the mm-wave analysis has revealed the 4 stable conformations theoretically predicted. The GS rotational constants were fitted at the $\mathrm{kHz}$ level of accuracy and the complete sets of quartic centrifugal distortion constants were determined. Moreover, pure rotational transitions in the low-frequency vibrational states were assigned and the degree of non-planarity of the MP may be discussed from the experimental inertial defects which are accentuated by the out of plane $-\mathrm{OCH}_{3}$ bending excitation. This study presents a similar approach on guaïacol (2-MP) and mequinol (4-MP) allowing to discuss the isomerism influence on the conformational landscape of methoxy-aromatic compounds.

\section{METHODS}

\section{A. Experimental issues}

Commercially available MP isomers (2-MP, 3-MP, and 4-MP) with stated purities higher than $98 \%$ were purchased from Acros Organics. Further purification was performed by continuous pumping for over $24 \mathrm{~h}$ in order to eliminate methanol traces. With room temperature equilibrium vapour pressures of around 10 and $140 \mu \mathrm{bar},{ }^{10} 2-\mathrm{MP}$ and 4 -MP are, respectively, the most and the less volatile MP isomers. The equilibrium MP vapour pressures were directly injected in the gas cell, and a continuous flow at a pressure of $10 \mu$ bar was maintained by a rough pumping.

The room temperature high resolution rotational spectra of the three isomers were measured in the $70-330 \mathrm{GHz}$ spectral range using two spectrometers of the Laboratory of Physical Chemistry of the Atmosphere (LPCA): line by line and broadband measurements were, respectively, performed with the absorption mm-wave spectrometer based on a frequency multiplication chain ${ }^{11}$ and the recently developed mm-wave chirped pulse Fourier transform (CPFT) spectrometer. ${ }^{12}$

\section{Frequency multiplication chain}

The sub-THz spectrometer based on a frequency multiplication chain was described in detail by Mouret et al. ${ }^{11}$ The molecules in gas phase are probed in a $1250 \mathrm{~mm}$-long and $56 \mathrm{~mm}$-diameter stainless steel absorption cell equipped with Teflon windows. The measurements were performed in a double pass configuration by adding a polarizing grid and a rooftop mirror. ${ }^{13,9}$ Two off-axis parabolic mirrors were placed, one before the grid to collimate the radiation into the cell and one after the grid to refocus it on the detector. A zero bias Schottky diode associated with a horn antenna and a liquid helium cooled InSb bolometer were used as detectors in the ranges 70-110 $\mathrm{GHz}$ and $140-330 \mathrm{GHz}$, respectively. A frequency modulation at $13 \mathrm{kHz}$ with a depth in the range of $300-900 \mathrm{kHz}$ was added to the mm-wave source in order to optimize the spectrometer sensitivity. The signal detection was performed by a standard lockin amplifier operating at the second harmonic of the modulation frequency. The individual frequency points were measured with an integration time of $50 \mathrm{~ms}$ or $200 \mathrm{~ms}$ depending on the absorption intensity with a frequency step of $100 \mathrm{kHz}$. The baseline arising from Fabry-Pérot effects presents strong amplitude variations and a free spectral range, both approximately 50 times broader than the molecular intensities and linewidths, respectively. These variations were easily removed from the spectra by using a low pass FFT filter as described in Ref. 49. The spectral resolution is limited to $100 \mathrm{kHz}$ since the observed lines are Doppler limited $\left[\Delta v_{\text {Doppler }} \approx 90 \mathrm{kHz}(\mathrm{FWHM})\right]$ and artificially broadened by the frequency modulation. ${ }^{11}$ The experimental error of measured line frequencies was estimated between $70 \mathrm{kHz}$ and 80 $\mathrm{kHz}$ using Eq. (2) of Ref. 14 taking into account the averaged S/N ratio, the frequency step and the molecular linewidth.

\section{Millimetre-wave chirped pulse Fourier transform spectrometer}

A CPFT spectrometer using an amplified multiplier chain covering the band from 190 to $210 \mathrm{GHz}$ was employed. This spectrometer is described in detail in Ref. 12. Briefly, the mm-wave CPFT spectrometer was used with a $1200 \mathrm{~mm}$-long stainless steel cell closed by Teflon windows. The mm-wave was collimated and propagated through the cell by two off-axis parabolic mirrors. The molecular re-emission beam is focused onto the detector by a Teflon lens. The CPFT spectrometer was used in segmented mode as described by Neill et al. ${ }^{15}: 23$ pulses of 700 ns duration and $1 \mathrm{GHz}$ bandwidth with an overlapping of $100 \mathrm{MHz}$ between each chirped pulse were used to cover the $20 \mathrm{GHz}$ band of the spectrometer. The free induction decay was recorded $20 \mathrm{~ns}$ after the end of the excitation pulse (recovery of the amplifier) and during $4 \mu \mathrm{s}$. Fast Fourier transform was employed on the full recorded data with application of Kaiser Bessel windows $(\beta=8)$ for side lobe suppression. The pressure was measured with a $1 \mathrm{mbar}$ full scale pressure gauge (MKS Baratron). All free induction decay signals have been measured at room temperature around $10 \mu$ bar. A comparison of the $\mathrm{S} / \mathrm{N}$ ratio obtained on 3-MP mm-wave spectra in the $200 \mathrm{GHz}$ frequency region with the two spectrometers is presented in Fig. 1. For a $1 \mathrm{GHz}$ range, the line by line absorption spectrum measured with the frequency multiplication chain and the emission broadband spectrum recorded 


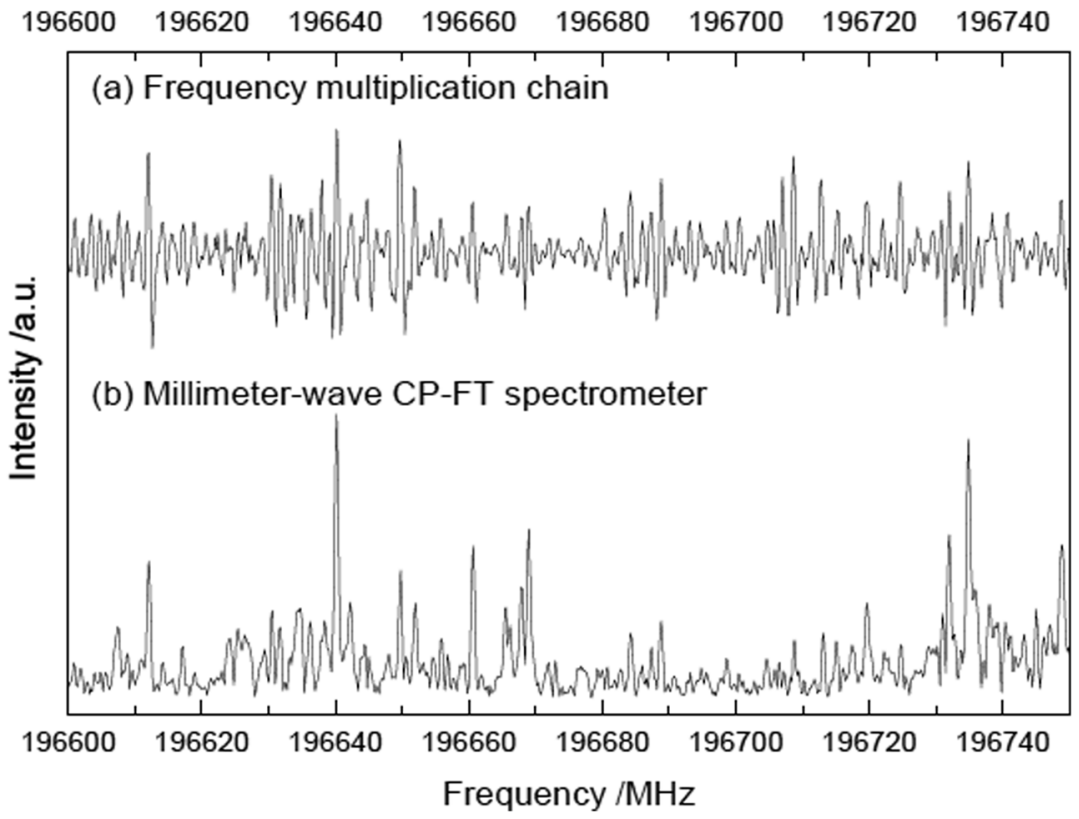

FIG. 1. Comparison between absorption (a) and emission (b) rotational spectra of 3-MP measured at room temperature in the $196.6 \mathrm{GHz}-196.75 \mathrm{GHz}$ frequency range with the frequency multiplication chain and the CPFT spectrometer, respectively. For the two spectra, the same acquisition time is used to record $1 \mathrm{GHz}$ ranged rotational spectra in line by line absorption measurements with frequency steps of $200 \mathrm{kHz}$ and time constant of $200 \mathrm{~ms}$ and broadband emission measurement with 16000000 averaged spectra.

with the CPFT instrument have been obtained with the same acquisition time by adjusting the frequency step to $200 \mathrm{kHz}$ and the time constant to $200 \mathrm{~ms}$ for the first spectrometer operating in $2 \mathrm{~F}$ modulation and by averaging 16000000 emission spectra for the second one. A slight but relevant improvement of the $\mathrm{S} / \mathrm{N}$ ratio $(\simeq \times 1.5)$ is obtained with the CPFT spectrometer. Then, experimental accuracies of the measured CPFT lines were estimated to $60 \mathrm{kHz}$ and $70 \mathrm{kHz}$, respectively, in the $190-210 \mathrm{GHz}$ spectral range.

TABLE I. Calculated and experimental GS parameters of observed conformers of guaïacol (2-MP C1) and cis- and trans-mequinol (4-MP C1 and C2): rotational constants, inertial defects $\Delta l=I_{c}-I_{b}-I_{a}$, and amplitude and orientation of the permanent dipole vector.

\begin{tabular}{|c|c|c|c|c|c|c|}
\hline & \multirow[b]{2}{*}{ Parameter } & \multirow[b]{2}{*}{ Unit } & \multicolumn{2}{|c|}{ Calculation: } & \multicolumn{2}{|c|}{ Experiment: } \\
\hline & & & $\begin{array}{c}\text { Reference } 6 \\
\text { B3LYP/6-31G(d,p) }\end{array}$ & $\begin{array}{c}\text { This work } \\
\text { B3LYP/cc-pVTZ }\end{array}$ & $\begin{array}{c}\text { Reference } 6 \\
\text { UV spectroscopy }\end{array}$ & $\begin{array}{c}\text { This work } \\
\text { mm-wave spectroscopy }\end{array}$ \\
\hline \multirow{6}{*}{ 2-MP C1 } & $A$ & $\mathrm{MHz}$ & 2609.8 & 2621.0 & 2607.1(1) & $2607.06260(117)$ \\
\hline & $B$ & $\mathrm{MHz}$ & 1549.8 & 1557.3 & $1560.8(1)$ & $1560.79645(40)$ \\
\hline & C & $\mathrm{MHz}$ & 978.4 & 983.0 & $982.9(1)$ & $982.871842(199)$ \\
\hline & $\Delta I$ & amu. $\AA^{2}$ & -3.20 & -3.22 & $-3.46(3)$ & $-3.45949(27)$ \\
\hline & $\mu$ & $\mathrm{D}$ & & 2.66 & & \\
\hline & $\angle(\vec{\mu}, a)$ & $\circ$ & & 37.5 & $28(1)^{b}$ & \\
\hline \multirow{6}{*}{ 4-MP C1 } & $A$ & $\mathrm{MHz}$ & 4888.8 & 4931.5 & $4880.2(1)$ & $4879.847983(75)$ \\
\hline & $B$ & $\mathrm{MHz}$ & 985.1 & 989.1 & $990.7(1)$ & $990.884961(33)$ \\
\hline & $C$ & $\mathrm{MHz}$ & 824.2 & 828.2 & $828.5(1)$ & $828.4395997(213)$ \\
\hline & $\Delta I$ & amu. $\AA^{2}$ & -3.22 & -3.22 & $-3.70(3)$ & $-3.555190(34)$ \\
\hline & $\mu$ & $\mathrm{D}$ & & 2.40 & & \\
\hline & $\angle(\vec{\mu}, a)$ & $\circ$ & & 89 & $88(1)^{b}$ & \\
\hline \multirow{6}{*}{ 4-MP C2 } & $A$ & $\mathrm{MHz}$ & 4881.9 & 4925.8 & $4871.7(1)$ & \\
\hline & $B$ & $\mathrm{MHz}$ & 985.6 & 989.5 & $991.5(1)$ & \\
\hline & C & $\mathrm{MHz}$ & 824.3 & 828.3 & $828.6(1)$ & \\
\hline & $\Delta I$ & amu. $\AA^{2}$ & -3.18 & -3.20 & $-3.53(2)$ & \\
\hline & $\mu$ & $\mathrm{D}$ & & 0.50 & & \\
\hline & $\angle(\vec{\mu}, a)$ & $\circ$ & & 27 & & \\
\hline
\end{tabular}

${ }^{a}$ Effective rotational constants (including terms due to quartic centrifugal distortion constants).

${ }^{\mathrm{b}}$ Electronic transition moment measured in Ref. 6. 


\section{B. Quantum chemical calculations}

High level of theory quantum chemistry calculations were performed using the Gaussian 16 package. ${ }^{16}$ As done for 3-MP, ${ }^{9}$ the potential energy surfaces (PES) of 2-MP and 4-MP were built at the B3LYP/6-311++G(d,p) level of theory by scanning with a step of $5^{\circ}$ the dihedral angles between the $-\mathrm{OH}$ and $-\mathrm{OCH}_{3}$ groups and the aromatic plane. Minima and saddle points were calculated with the B3LYP functional and larger Dunning ${ }^{17}$ correlation consistent basis set cc-pVTZ with an optimisation process using the tight convergence criteria providing the GS energies, rotational constants, dipole moments of the stable conformers and conformational barriers (see Table I). The B3LYP/cc-pVTZ level of theory has proved one's worth for the computational study on the molecular conformations of phenolic compounds. ${ }^{18}$ Anharmonic frequencies have been computed with the same level of theory using an ultrafine grid. Finally, the MOLPRO program, ${ }^{19}$ and the Multifunctional Wavefunction Analyser (Multiwfn) ${ }^{20}$ have been used to evidence the formation of intramolecular H-Bond in 2-MP: with MOLPRO, the 2-MP molecule was optimized in its GS and its wave function generated at the cc-pVDZ level of theory; subsequently, the Multifwn code has been employed to extract the electron density from the corresponding wave function in order to analyse its topology, highlighting critical structures that evidenced, from the standpoint of the quantum theory of atom in molecules, the formation of an intramolecular H-bond.

\section{RESULTS AND DISCUSSION}

\section{A. Theoretical analysis}

\section{Guaïacol (ortho-, 2-MP)}

Figure 2 presents the PES built at the B3LYP/6-311++G(d,p) level of theory describing the conformational space of 2-MP. A deep minimum is observed when the $\mathrm{O}-\mathrm{H}$ and the $\mathrm{O}-\mathrm{CH}_{3}$ bonds lie in the benzene ring plane with the $\mathrm{H}$ atom of the phenol group in the donor position between the two oxygen atoms. This conformer labelled $\mathrm{C} 1$ is stabilized by the intramolecular $\mathrm{O}-\mathrm{H} \cdots \mathrm{OCH}_{3}$ $\mathrm{HB}$ indirectly evidenced via an electronic origin red-shifted compared to phenol in the vibrationally resolved enhanced multiphoton ionization (REMPI) spectrum of Ref. 6 and in the core photoelectron spectra (XPS) of Ref. 4 indicating that the high energy conformers of 2-MP show significant changes when the $\mathrm{HB}$ is broken. These higher energy conformers labelled C2 and C3 are identified as minima of the 2-MP PES (Fig. 2) with strongly higher energies compared to C1 (energy differences of $1392 \mathrm{~cm}^{-1}$ and $1720 \mathrm{~cm}^{-1}$ are, respectively, calculated for $\mathrm{C} 2$ and $\mathrm{C} 3$ at the B3LYP/cc-pVTZ level of theory). In agreement with the optimised rotamer structures calculated by Islam et al. ${ }^{4}$ with a smaller basis set, the $\mathrm{C} 2$ conformer exhibits the $\mathrm{O}-\mathrm{H}$ and the $\mathrm{O}-\mathrm{CH}_{3}$ bounds lying in the $a b$ plane but with the hydroxyl group turned $180^{\circ}$ compared to $\mathrm{C} 1$. In $\mathrm{C} 3$, the $\mathrm{O}-\mathrm{CH}_{3}$ bond is out of plane and this higher energy conformer is stabilised by a $\mathrm{H}-\mathrm{O} \cdots \mathrm{HCH}_{2} \mathrm{O}$ longer than the $\mathrm{O}-\mathrm{H} \cdots \mathrm{OCH}_{3} \mathrm{HB}$ in $\mathrm{C} 1$. Two

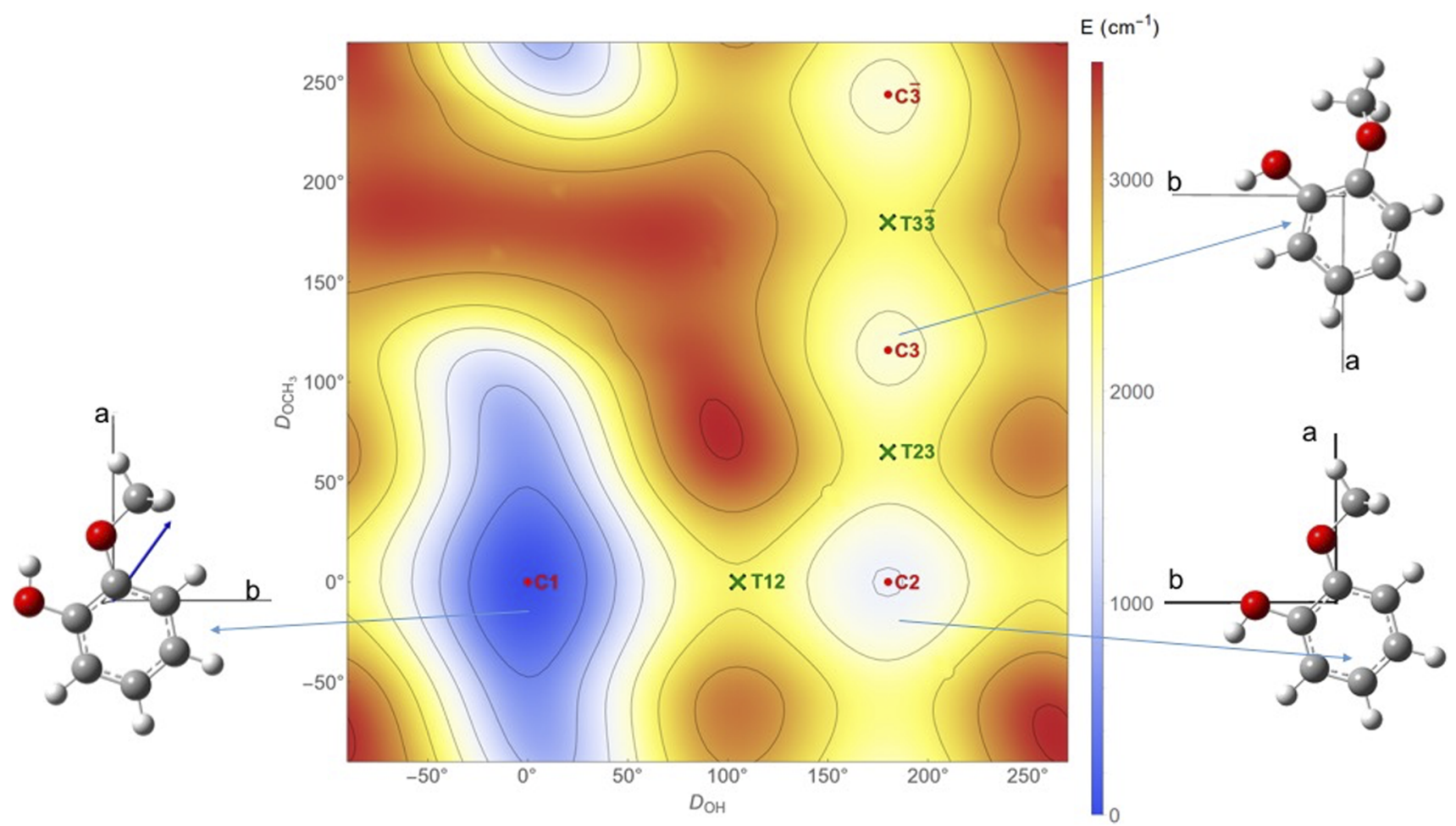

FIG. 2. PES describing the conformational space of 2-MP built with a step of $5^{\circ}$ at the B3LYP/6-311++G(d,p) level of theory. Local minima and saddle points are, respectively, marked with a red dot and a green cross. Geometries of $\mathrm{C} 1, \mathrm{C} 2$, and C3 stable conformers are optimized at the B3LYP/cc-pVTZ level of theory shown in the ab plane with the permanent dipolar vector (blue arrow) for C1. 
isoenergetic minima C3 and its mirror image C3 are observed in the PES depending on the positions up and down of the methoxy $\mathrm{O}-\mathrm{CH}_{3}$ group in relation to the ab plane. The rotamers $\mathrm{C} 2, \mathrm{C} 3$, and C3 are too high in energy to be observed at room temperature and only the most stable $\mathrm{C} 1$ is expected in the mm-wave spectrum. For this conformer, $a$-type and $b$-type rotational transitions are expected since the permanent dipole moment is oriented in the ab plane with the $\left|\mu_{a}\right|$ and $\left|\mu_{b}\right|$ components estimated, respectively, to 2.1 and 1.6 Debye at the B3LYP/cc-pVTZ level of theory (Table I).

In order to characterize the intramolecular $\mathrm{O}-\mathrm{H} \cdots \mathrm{OCH}_{3} \mathrm{HB}$ in $\mathrm{C} 1$, a topology analysis for electron density was performed following the method described in Sec. II B. The results are presented in Fig. 3 where we have considered the critical points of the positive electron density distribution function of 2-MP. In addition to covalent bonds drawing the 2-MP C1 conformer, the intramolecular $\mathrm{O}-\mathrm{H} \cdots \mathrm{OCH}_{3}$ HB appears clearly. The Multifwn analysis shows that the covalent bond critical points have a potential energy density between -0.357 and $-0.798 \mathrm{E}_{h}$, while the $\mathrm{O}-\mathrm{H} \cdots \mathrm{OCH}_{3}$ bond has a potential energy density of $-0.022 \mathrm{E}_{h}$, significantly weaker than the covalent energies. This value is characteristic of a moderate intramolecular $\mathrm{HB}$, as it was demonstrated by the computational study of Cesari et al. in Ref. 18.

\section{Mequinol (para-, 4-MP)}

The PES of 4-MP shown in Fig. 4 exhibits four minima associated with four stable rotamers $\mathrm{C} 1, \overline{\mathrm{C}} 1, \mathrm{C} 2$, and $\overline{\mathrm{C}} 2$. Considering the mirror reflexion in a symmetry plane containing the c-axis and the two $\mathrm{C}-\mathrm{O}$ bonds, $\mathrm{C} 1$ and $\mathrm{C} 2$ are, respectively, equivalent to $\overline{\mathrm{C}} 1$ and

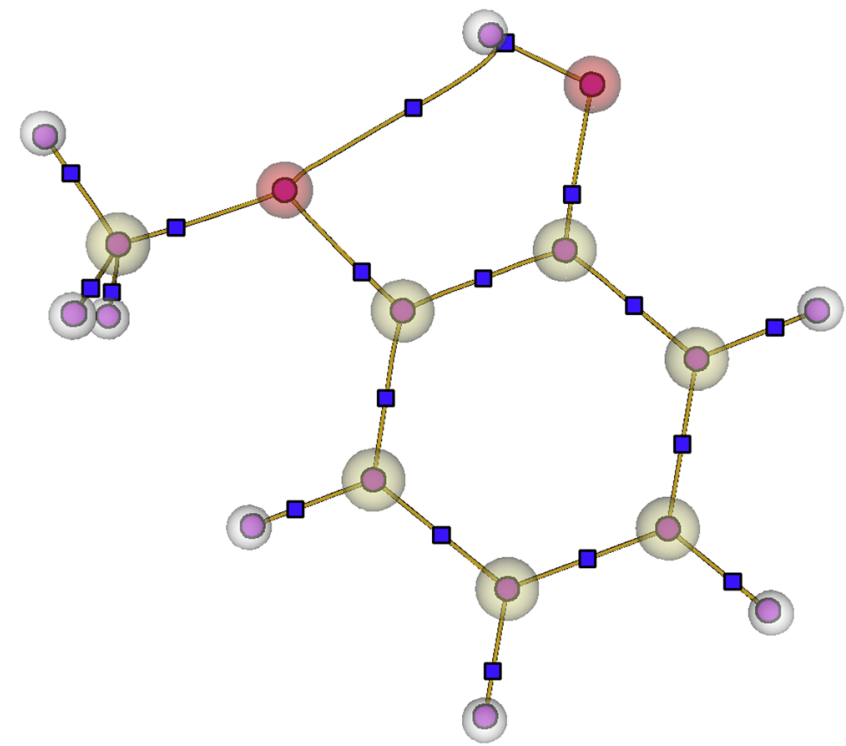

FIG. 3. Topology analysis for electron density on 2-MP C1 optimized at the CCSD/cc-pVDZ level of theory using the Multifunctional Wavefunction Analyser (Multiwfn). ${ }^{20}$ The full circles, observed at the center of the atoms, represent the atomic critical points corresponding to local maxima in the three perpendicular directions of spaces. The full squares correspond to saddle points (maximum in two directions of space and minimum in the third one) of electron density. The connections between maxima of the electron density via the saddle points are represented with full squares.
C2 with isoenergetic PES minima. C1 and C2 correspond, respectively, to the cis- and trans-rotamer of 4-MP. An energy difference $\Delta E=E_{C 1}-E_{C 2}$ was estimated to $5.7 \mathrm{~cm}^{-1}$ with the B3LYP/ccpVTZ level of theory calculations. Islam et al. in Ref. 4 have obtained $\Delta E=1.4 \mathrm{~cm}^{-1}$ with a smaller $6-311++\mathrm{G}^{* *}$ basis-set. For both cases, taking into account the accuracy of density functional theory (DFT) calculations, the difference is too small to be significant and the $\mathrm{C} 1$ and $\mathrm{C} 2$ conformers of 4-MP may be considered as isoenergetic with equivalent populations in the conformational mixture. The saddle points noted T12 in Fig. 4, calculated around $890 \mathrm{~cm}^{-1}$ at the B3LYP/cc-pVTZ level of theory, indicate that the conformational barriers are too high to observe tunneling splitting between $\mathrm{C} 1$ and $\mathrm{C} 2$ rotamers. As shown in Table I with the GS calculated parameters, the rotational constants and therefore the inertial defects of cis- and trans-mequinol are very close, whereas their permanent dipoles are strongly different. The module of the $\mathrm{C} 1$ permanent dipole moment is 4.8 times longer than the one of $\mathrm{C} 2$. $a$-type and $b$-type rotational transitions are predicted for $\mathrm{C} 2$ whereas only strong $b$-type transitions are expected for $\mathrm{C} 1$. Consequently, the observation of a $b$-type rotational $\mathrm{mm}$-wave spectrum for 4 -MP in -cis configuration is privileged in comparison with an hypothetical observation of the -trans 4-MP $a$-type and $b$-type rotational signatures. In Ref. 6, Ruiz-Santoyo et al. were able to assign individually rotationally resolved electronic spectra in the $S_{1}$ state of the -cis and -trans 4-MP rotamers by exciting the two electronic origin bands separated by around $97 \mathrm{~cm}^{-1}$ on the one-color REMPI spectrum.

\section{B. Spectral analysis}

Spectral analysis was performed using SPFIT/SPCAT Pickett's programs $^{21}$ where a Watson-type Hamiltonian, describing a semirigid rotor model developed up to quartic centrifugal distortion constants, is implemented. We have chosen, in $I^{r}$ representation, the A-reduction for 2-MP and the S-reduction for 4-MP since 4MP is nearly prolate $(\kappa \simeq-0.92)$, whereas 2 -MP is strongly asymmetric $(\kappa \simeq-0.3)$. The AABS (Assignment and Analysis of Broadband Spectra) package from Kisiel ${ }^{22,23}$ was used for a direct assignment of measured lines to predicted rotational transitions. As for $3-\mathrm{MP},{ }^{9}$ a specific treatment of the internal rotation of the methyl group was not required in this study. Indeed, the barrier heights hindering the internal rotation for the MP isomers are very high (around $900 \mathrm{~cm}^{-1}$ ) and the internal rotation splitting could not be resolved as in several cases of methoxy groups attached to benzene rings such as methyl anisole isomers. ${ }^{24-26}$ Pure rotational transitions in the low-energy vibrationally excited states (ES) (typically below $\left.400 \mathrm{~cm}^{-1}\right)^{27}$ were observed in the room-temperature mmwave spectra. Therefore an analysis of the ES of 2-MP and 4-MP was undertaken subsequent to the GS analysis.

\section{GS analysis}

The GS rotational transitions of 2-MP were first assigned by comparing the experimental spectrum to the preliminary prediction obtained with the SPCAT program with the pure rotational constants measured in Ref. 6 and the quartic centrifugal distortion constants resulting from our quantum chemical frequency calculations including the analysis of the vibrational-rotational coupling. A $15 \mathrm{GHz}$ portion of the measured mm-wave spectra highlighting the huge density of lines and the results of the spectral analysis of 


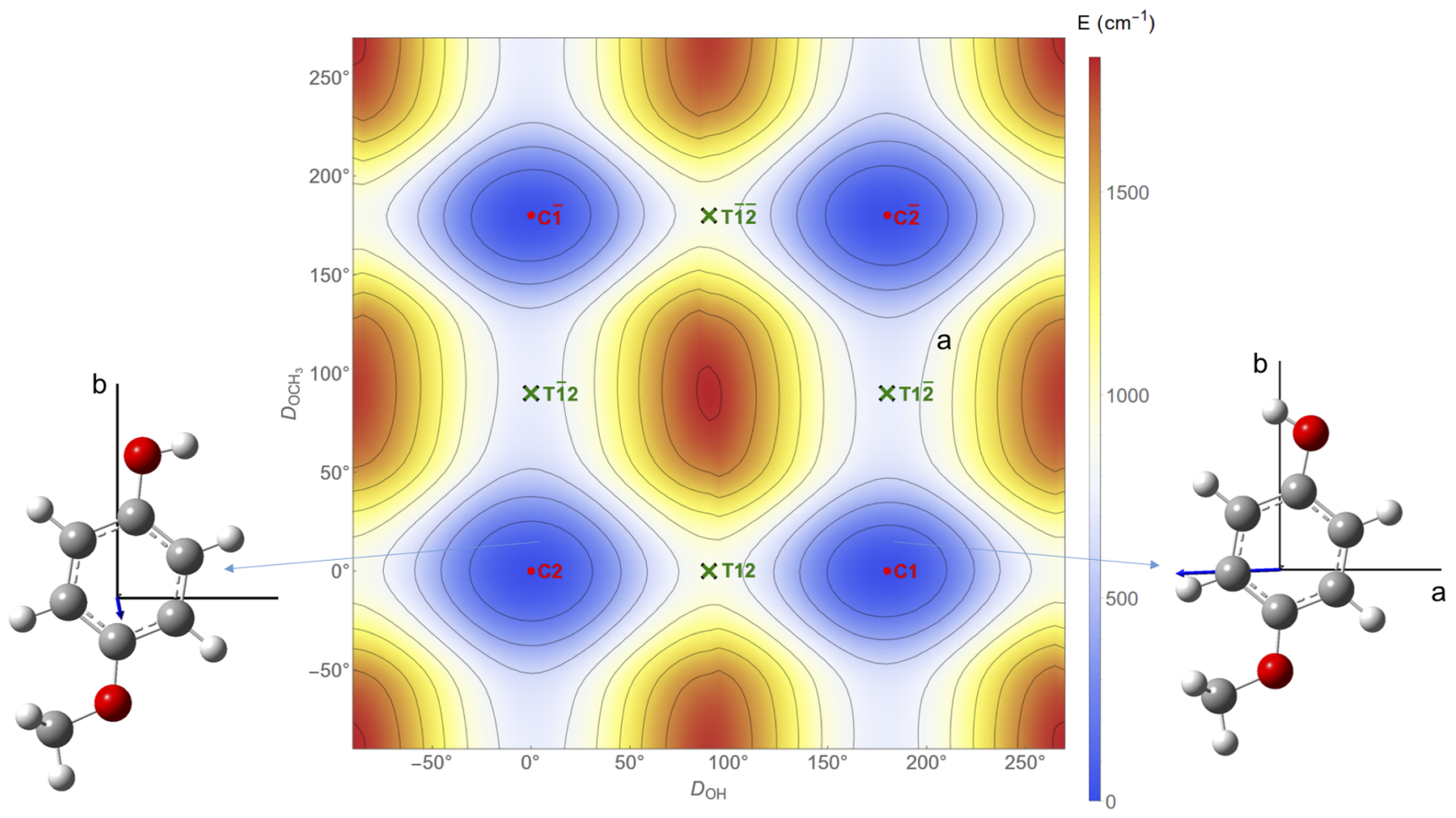

FIG. 4. PES describing the conformational space of 4-MP built with a step of $5^{\circ}$ at the B3LYP/6-311++G(d,p) level of theory. Local minima and saddle points are, respectively, marked with a red dot and a green cross. Geometries of $\mathrm{C} 1$ and $\mathrm{C} 2$ stable conformers are optimized at the B3LYP/cc-pVTZ level of theory shown in the ab plane with their permanent dipolar vectors (blue arrow).

2-MP is presented in Fig. 5. In agreement with the previous theoretical considerations, only the conformer $\mathrm{C} 1$, stabilised by the intramolecular $\mathrm{HB}$, was observed in the rotational spectrum of 2-MP measured between 70 and $330 \mathrm{GHz}$. Starting from the SPCAT simulations from the initial set of molecular parameters, the fit was iteratively extended up to $J$ and $K_{a}$ values equal to 167 and 79,
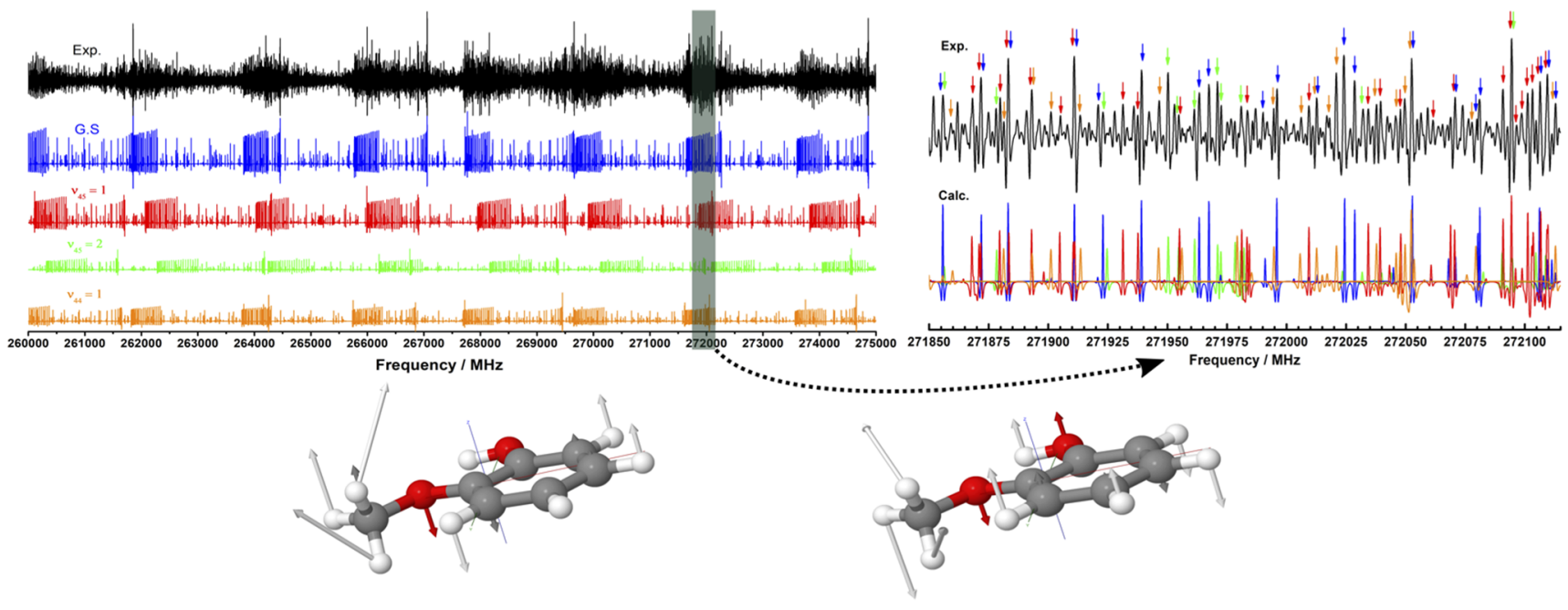

$v_{45}: \mathrm{OCH}_{3}$ out of plane bending

$\mathrm{v}_{44}: \mathrm{CH}_{3}$ torsion

FIG. 5. Top: The experimental (in black) mm-wave spectrum (left: $260-275 \mathrm{GHz}$ scan, right: zoom of $250 \mathrm{MHz}$ ) of 2-MP C1 is compared with the fitted spectra in color (GS in blue, vibrationally ES $v_{45}=1, v_{45}=2$ and $v_{44}=1$ in red, green and orange, respectively). Bottom: description of the $v_{45}$ and $v_{44}$ vibrational modes. 
TABLE II. Summary of the fitted parameters for 2-MP C1 and 4-MP C1 in the GS. The number N of assigned rotational lines in the fit as well as the maximum values of quantum numbers $\left(J_{\max }, K_{\max }\right)$ and the standard deviations are indicated.

\begin{tabular}{|c|c|c|c|c|c|}
\hline Parameter & Unit & $\begin{array}{c}\text { This work mm-wave } \\
\text { spectroscopy }\end{array}$ & Parameter & Unit & $\begin{array}{l}\text { This work mm-wave } \\
\text { spectroscopy }\end{array}$ \\
\hline \multicolumn{3}{|c|}{ 2-MP C1 } & \multicolumn{3}{|c|}{ 4-MP C1 } \\
\hline$A$ & $\mathrm{MHz}$ & $2607.06260(117)$ & $A$ & $\mathrm{MHz}$ & $4879.847983(75)$ \\
\hline$B$ & $\mathrm{MHz}$ & $1560.79645(40)$ & $B$ & $\mathrm{MHz}$ & $990.884961(33)$ \\
\hline$C$ & $\mathrm{MHz}$ & $982.871842(199)$ & $C$ & $\mathrm{MHz}$ & $828.4395997(213)$ \\
\hline$\Delta_{J}$ & $\mathrm{kHz}$ & $0.0547088(185)$ & $D_{J}$ & $\mathrm{kHz}$ & $0.02006090(102)$ \\
\hline$\Delta_{K}$ & $\mathrm{kHz}$ & $0.192821(310)$ & $D_{K}$ & $\mathrm{kHz}$ & $0.987496(34)$ \\
\hline$\Delta_{J K}$ & $\mathrm{kHz}$ & $0.063713(200)$ & $D_{J K}$ & $\mathrm{kHz}$ & $-0.0374643(65)$ \\
\hline$\delta_{J}$ & $\mathrm{~Hz}$ & $19.8112(89)$ & $d_{1}$ & $\mathrm{~Hz}$ & $-4.19403(59)$ \\
\hline$\delta_{K}$ & $\mathrm{~Hz}$ & $111.528(86)$ & $d_{2}$ & $\mathrm{~Hz}$ & $-0.341409(228)$ \\
\hline $\mathrm{N}_{a-t y p e}$ & & 6657 & $\mathrm{~N}_{a-\text { type }}$ & & 0 \\
\hline $\mathrm{N}_{b-t y p e}$ & & 5215 & $\mathrm{~N}_{b-t y p e}$ & & 6076 \\
\hline$J_{\max }$ & & 167 & $J_{\max }$ & & 196 \\
\hline$K_{\max }$ & & 79 & $K_{\max }$ & & 42 \\
\hline $\mathrm{rms}$ & $\mathrm{kHz}$ & 88.2 & $\mathrm{rms}$ & $\mathrm{kHz}$ & 83.1 \\
\hline Unitless rms & & 1.02 & Unitless rms & & 1.05 \\
\hline
\end{tabular}

respectively. In total, around 12000 lines were assigned to GS rotational transitions shared as $a$ - and $b$-type according to the calculated dipole moment orientation. Pure rotational constants $A, B$, and $C$ were carried out at the $\mathrm{kHz}$ level with higher accuracy compared to Ref. 6 and the complete set of quartic centrifugal distortion constants $\left(\Delta_{J}, \Delta_{K}, \Delta_{J K}, \delta_{J}\right.$, and $\left.\delta_{K}\right)$ were well determined to reproduce the measured transitions at the experimental accuracy (see Table II).

The same assignment procedure was undertaken for 4-MP. Nevertheless, compared to 2-MP, the assignment of 4-MP spectra was more difficult due, in part, to lower rotational absorptions/emissions essentially explained by a room temperature equilibrium vapour pressure 15 times weaker. ${ }^{10}$ An example of 4-MP $\mathrm{mm}$-wave rotational spectrum and its assignment is presented in Fig. 6. As it was expected by the theoretical analysis (see Sec. III A), only the cis-rotamer (4-MP C1) was assigned in the experimental spectra and the trans conformation (4-MP C2) was not observed. Indeed, in addition to the low vapour pressure of 4-MP, the weak polarity of the trans-rotamer (see Table I: $\left(\frac{\mu_{b, 4-M P C 1}}{\mu_{a, 4-M P C 2}}\right)^{2} \simeq 30$ ) prevents its observation in our experimental conditions. Therefore, the 4-MP mm-wave spectra exhibit only rotational transitions of the cis-rotamer (4-MP C1). Starting from the scaled B3LYP/cc-pVTZ rotational constants, ${ }^{28}$ the GS $b$-type rotational transitions of 4-MP $\mathrm{C} 1$ were straightforwardly assigned and its molecular parameters fitted at the experimental accuracy (see Table II). Compared to 2-MP, the same degree of accuracy is reached on the rotational and quartic centrifugal distortion constants with more than $6000 b$-type transitions assigned and a global rms close to $80 \mathrm{kHz}$. Even with the observation of a unique conformer, a lot of lines remain unassigned after the GS analysis, which indicates the large contribution of pure rotational transitions in the vibrational ES.

\section{Vibrationally ES analysis}

In the case of 2-MP, following the successful analysis of the GS, numerous remaining lines were assigned to pure rotational transitions in the vibrationally ES populated at room temperature. Three sets of ES molecular parameters were included in a global fit procedure to assign all the rotational structures by comparing the experimental and calculated spectra from the fitted parameters (Fig. 5). The assigned vibrationally ES are located below $200 \mathrm{~cm}^{-1}$ and correspond to the three lowest energy vibrations. Using the FT FarIR synchrotron middle-resolution measurements of Cuisset et al. in Ref. 29 and the B3LYP/cc-pVTZ anharmonic frequencies of the 2-MP C1 rotamer, the three lowest vibrational levels have been identified: the first level is the fundamental $\mathrm{v}_{45}=1$ methoxy out of plane bending; the second level is its overtone $\mathrm{v}_{45}=2$ and the third level is the methyl torsion fundamental $\mathrm{v}_{44}=1$ (experimental and calculated harmonic and anharmonic frequencies are listed in Table III). An initial set of spectroscopic parameters for these ES were calculated from calculated anharmonic corrections and scaled with a factor corresponding to the ratio between experimental and calculated rotational constants in the GS. ${ }^{28}$ All the fitted spectroscopic parameters resulting from the global fit are summarized in Table III. As for the GS, pure rotational constants were carried out at the $\mathrm{kHz}$ level and the complete set of quartic centrifugal distortion constants were well determined to reproduce the measured transitions at the experimental accuracy. Near 20000 lines of the 2-MP spectra have been assigned to $a$-type and $b$-type pure rotational transitions in the low-energy vibrationally ES.

The vibrationally ES analysis of 4-MP C1 was performed identically to 2-MP C1. As for 2-MP, pure rotational transitions in the $\mathrm{V}_{45}=1, \mathrm{v}_{45}=2$, and $\mathrm{v}_{44}=1$ were identified. The results are presented in the second part of Table III. Unlike 2-MP, the lowest energy 

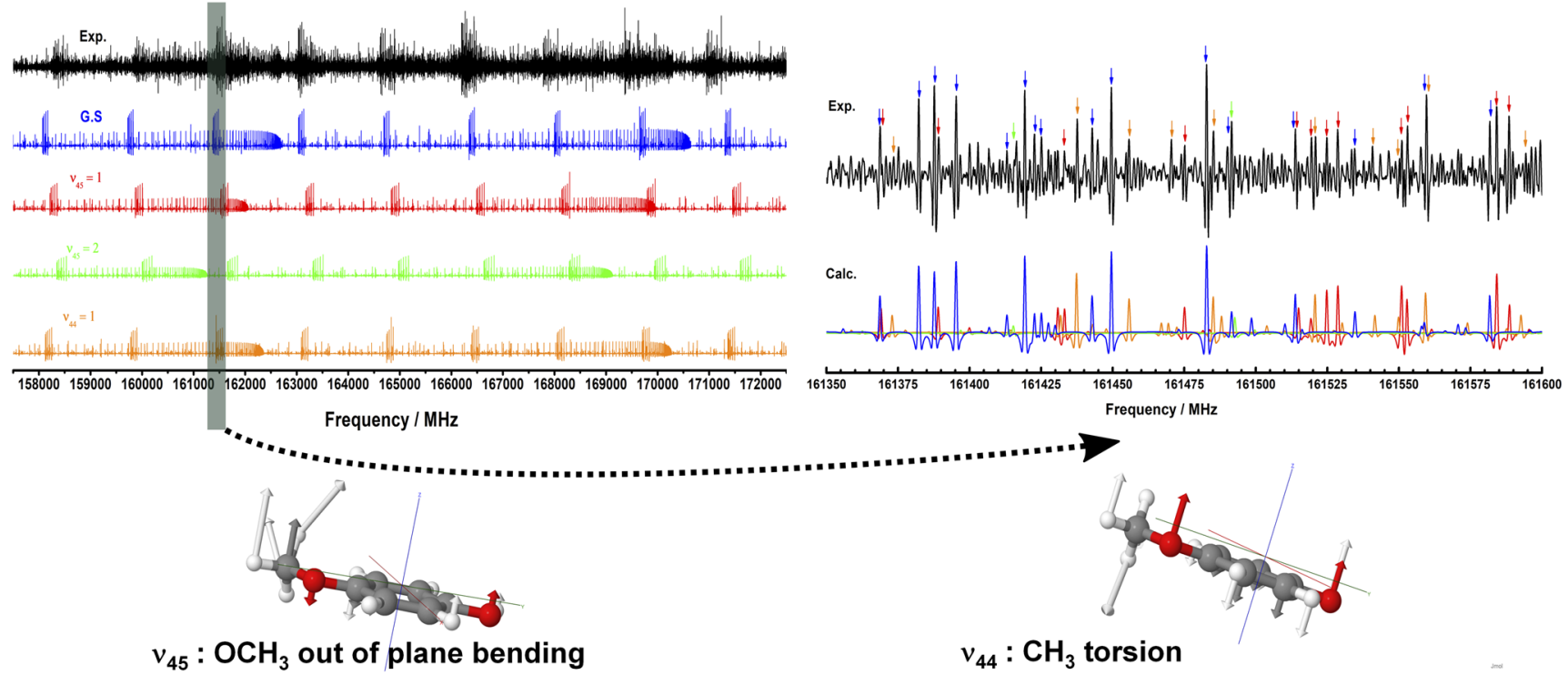

FIG. 6. Top: The experimental (in black) mm-wave spectrum (left: $157-173 \mathrm{GHz}$ scan, right: zoom of $250 \mathrm{MHz}$ ) of 4-MP C1 is compared with the fitted spectra in color (GS in blue, vibrationally ES $v_{45}=1, v_{45}=2$, and $v_{44}=1$ in red, green and orange, respectively). Bottom: description of the $v_{45}$ and $v_{44}$ vibrational modes.

vibrational band of 4-MP was not observed in the FT-Far-IR gas phase spectra of Ref. 27. The B3LYP/cc-pVTZ anharmonic frequency calculation has predicted the $v_{45}$ fundamental mode at $66.5 \mathrm{~cm}^{-1}$ red-shifted compared to the one of 2-MP C1 whereas the $v_{44}$ of 4-MP is blue-shifted. As in 2-MP, all the rotational constants in $\mathrm{v}_{45}=1 \mathrm{ES}$ were correctly determined with a comparable degree of accuracy. For $\mathrm{v}_{45}=2$ and $\mathrm{v}_{44}=1$, weaker intensity rotational lines complicate the assignment and it was not possible to reach the same fit quality with larger values of the rms. Compared to the other vibrationally ES analyses, we cannot exclude here a possible overlap of the rotational structures of $\mathrm{v}_{45}=2$ and $\mathrm{v}_{44}=1$ taking into account the rather close vibrational frequencies calculated at the $\mathrm{B} 3 \mathrm{LYP} / \mathrm{cc}-$ pVTZ level of theory. In our case, the isolated state model turned out to be adequate within the accuracy of our data for 2-MP and 4-MP except for the two previously mentioned states. A specific treatment of the perturbation by the introduction of interaction terms is in this case required if a sufficient level mixing is reached. ${ }^{41}$ The energy file generated by the SPCAT Pickett's program ${ }^{21}$ provides us the mixing coefficients $P_{\text {mix }}$ of 4-MP rotational transitions in the $\mathrm{v}_{45}=2$ and $\mathrm{v}_{44}=1$ ES. Figure 7 shows the evolution of the mixing coefficient with the $J^{\prime \prime}$ and $K_{a}{ }^{\prime \prime}$ quantum numbers for $\mathrm{v}_{45}=2$ (red circles) and $\mathrm{v}_{44}=1$ (black circles) ES. This figure highlights a strong perturbation between the two ES with important level mixing $\left(\mathrm{P}_{\text {mix }}>5 \%\right)$ for high $J^{\prime \prime}$ and $K_{a}^{\prime \prime}$ values $\left(J^{\prime \prime} \simeq K_{a}^{\prime \prime}>120\right)$. In our case, due to a limited sensitivity, only transitions with $K_{a}^{\prime \prime}<34$ may be assigned and it was not possible to improve the quality of the fit by adding perturbation parameters such as $b$-type Coriolis interaction terms in the global fit.

\section{Inertial defects}

The inertial defect $\Delta I$ in amu. $\AA^{2}$ units is a molecular parameter directly determined from the principal moments of inertia
$I_{\alpha}(\alpha=a, b, c)$ or from the rotational constants $A, B, C$ in $\mathrm{MHz}$ using the relation

$$
\Delta I=I_{c}-I_{b}-I_{a}=\frac{h}{8 \pi^{2}}\left(\frac{1}{C}-\frac{1}{B}-\frac{1}{A}\right)
$$

with $\frac{h}{8 \pi^{2}}=5.05379 \times 10^{5}$ amu. $\AA^{2} . M H z . \Delta I$ is very nearly 0 for a planar molecule and is negative for non-planar molecules. ${ }^{42}$ Therefore, the value of the inertial defect is in general used in rotationally resolved spectroscopy to probe the degree of non-planarity of a molecule.

\section{GS inertial defects}

Several groups worked on the determination of a general formula providing the inertial defect of planar molecules with very low out-of-plane vibrations: in particular, Morino and Oka have generalised the expression of $\Delta I$ for any planar asymmetric top. ${ }^{43}$ All these studies provided theoretical values of the GS $\Delta I$ in very good agreement with rotationally resolved measurements. Moreover, with the increasing quantity of high-resolution GS data on benzene derivatives and polycyclic aromatic compounds exhibiting a slight negative inertial defect, several authors have determined semi-empirical relations between the inertial defect $\Delta I$, the wavenumber of the lowest out-of plane vibration $v_{l}$, and the square root of the $c$-axis principal moment of inertia $\sqrt{I_{c}} \cdot 42,44,45$

In the context of the MP study, we have identified in the literature GS rotationally resolved measurements on phenol and anisole derivatives for which all isomers were studied. Table IV compares the GS inertia defects of ortho, meta, and para isomers of phenol and anisole derivatives in order to explain negative inertial defects of methoxyphenol isomers. The main contribution to the non-planarity and to the negative $\Delta I$ of $\mathrm{MP}$ comes from the out of plane hydrogen atoms in the methoxy 
TABLE III. Summary of the fitted parameters in the vibrationally ES for 2-MP C1 and 4-MP C1. Experimental vibrational wavenumbers from Ref. 29 and rotational constants fitted in this study are compared with B3LYP/cc-pVTZ anharmonic calculations. The number $\mathrm{N}$ of assigned rotational lines in the fit as well as the maximum values of quantum numbers $\left(J_{\max }, K_{\max }\right)$ and the standard deviations are indicated.

\begin{tabular}{|c|c|c|c|c|c|c|c|}
\hline & Parameter & Expt. & Calc. & Expt. & Calc. & Expt. & Calc. \\
\hline \multirow{18}{*}{ 2-MP C1 } & & \multicolumn{2}{|l|}{$\mathrm{v}_{45}=1$} & \multicolumn{2}{|l|}{$\mathrm{V}_{45}=2$} & \multicolumn{2}{|l|}{$\mathrm{v}_{44}=1$} \\
\hline & $v_{\text {harm }} / \mathrm{cm}^{-1}$ & & 77.0 & & 154.0 & & 178.6 \\
\hline & $v_{\text {anharm }} / \mathrm{cm}^{-1}$ & 63.5 & 76.2 & & 152.0 & & 105.8 \\
\hline & $A / \mathrm{MHz}$ & $2605.45291(106)$ & 2604.72 & $2604.06862(201)$ & 2603.43 & $2604.9992(34)$ & 2603.46 \\
\hline & $B / \mathrm{MHz}$ & $1560.52461(58)$ & 1541.35 & $1560.17805(246)$ & 1540.90 & $1559.26902(226)$ & 1540.27 \\
\hline & $\mathrm{C} / \mathrm{MHz}$ & $984.254416(198)$ & 976.45 & $985.681371(312)$ & 977.77 & $983.12326(35)$ & 975.34 \\
\hline & $\Delta I / \mathrm{amu} . \AA^{2}$ & $-4.35793(30)$ & -4.339 & $-5.27632(82)$ & -5.229 & $-4.06172(91)$ & -4.074 \\
\hline & $\Delta_{J} / \mathrm{kHz}$ & $0.055918(41)$ & & $0.057177(82)$ & & $0.055106(264)$ & \\
\hline & $\Delta_{K} / \mathrm{kHz}$ & $0.194528(245)$ & & $0.19265(34)$ & & $0.18457(101)$ & \\
\hline & $\Delta_{J K} / \mathrm{kHz}$ & $0.066908(225)$ & & $0.07195(33)$ & & $0.06721(114)$ & \\
\hline & $\delta_{J} / \mathrm{Hz}$ & $20.1149(199)$ & & $20.384(42)$ & & $19.910(131)$ & \\
\hline & $\delta_{K} / \mathrm{Hz}$ & $113.784(115)$ & & $115.327(216)$ & & $111.69(57)$ & \\
\hline & $\mathrm{N}_{a-t y p e}$ & 5051 & & 2766 & & 1930 & \\
\hline & $\mathrm{N}_{b-t y p e}$ & 4144 & & 2866 & & 2023 & \\
\hline & $J_{\max }$ & 166 & & 166 & & 165 & \\
\hline & $K_{\max }$ & 88 & & 60 & & 60 & \\
\hline & $\mathrm{rms} / \mathrm{kHz}$ & 92.6 & & 89.6 & & 83.0 & \\
\hline & Unitless rms & 1.13 & & 1.14 & & 1.21 & \\
\hline \multirow{18}{*}{ 4-MP C1 } & & \multicolumn{2}{|l|}{$\mathrm{v}_{45}=1$} & \multicolumn{2}{|l|}{$\mathrm{v}_{45}=2$} & \multicolumn{2}{|l|}{$\mathrm{v}_{44}=1$} \\
\hline & $v_{\text {harm }} / \mathrm{cm}^{-1}$ & & 81.0 & & 162.0 & & 156.2 \\
\hline & $v_{\text {anharm }} / \mathrm{cm}^{-1}$ & & 66.5 & & 148.7 & & 127.5 \\
\hline & $A / \mathrm{MHz}$ & $4864.772323(86)$ & 4887.01 & 4846.49574(59) & 4870.67 & $4872.25201(111)$ & 4892.73 \\
\hline & $B / \mathrm{MHz}$ & $991.039897(48)$ & 980.50 & $991.25815(50)$ & 980.74 & $990.70800(90)$ & 980.05 \\
\hline & $\mathrm{C} / \mathrm{MHz}$ & $829.410452(33)$ & 822.57 & $830.396581(57)$ & 823.50 & $828.849041(81)$ & 821.94 \\
\hline & $\Delta I / \mathrm{amu} . \AA^{2}$ & $-4.510463(51)$ & -4.452 & $-5.51354(31)$ & -5.366 & -4.109 10(55) & -4.0971 \\
\hline & $D_{J} / \mathrm{kHz}$ & $0.02046653(163)$ & & $0.0206232(113)$ & & $0.0206123(158)$ & \\
\hline & $D_{K} / \mathrm{kHz}$ & $1.005240(42)$ & & $0.98844(38)$ & & $1.78337(100)$ & \\
\hline & $D_{J K} / \mathrm{kHz}$ & $-0.0409777(85)$ & & $-0.052632(75)$ & & $-0.057969(155)$ & \\
\hline & $d_{1} / \mathrm{Hz}$ & $-4.19873(82)$ & & $-4.0490(78)$ & & $-4.3334(109)$ & \\
\hline & $d_{2} / \mathrm{Hz}$ & $-0.32290(36)$ & & $-0.30507(198)$ & & $-0.2538(33)$ & \\
\hline & $\mathrm{N}_{a-t y p e}$ & 0 & & 0 & & 0 & \\
\hline & $\mathrm{N}_{b-t y p e}$ & 4508 & & 1093 & & 965 & \\
\hline & $J_{\max }$ & 188 & & 190 & & 175 & \\
\hline & $K_{\max }$ & 41 & & 35 & & 27 & \\
\hline & $\mathrm{rms} / \mathrm{kHz}$ & 91.5 & & 123.8 & & 139.6 & \\
\hline & Unitless rms & 1.16 & & 1.57 & & 1.78 & \\
\hline
\end{tabular}

group, while the $-\mathrm{OH}$ group does not induce a significant negative inertial defect. Therefore, the $\Delta I$ values of MP are slightly lower than the $\Delta I$ of $\mathrm{cresol}^{36-38}$ and anisole. ${ }^{39}$ The $\Delta I$ values

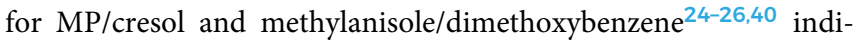
cate a stronger contribution on the negative inertial defects for the methoxy group compared to the methyl group, probably explained by the contribution of the $\mathrm{OCH}_{3}$ out of plane contribution $\left(v_{l}=v_{45}\right.$ for MP) leading to a slight negative zero point inertial defect. $^{44}$

Now, when we focus our attention on the isomeric dependence on the inertial defects, the most negative value is always obtained for the para isomer and the $\Delta I$ value of the ortho isomer appears to be slightly lower than that of the meta isomer. From these observations, two complementary elements of explanation may be proposed: (i) the zero point inertial defect induced by the low-energy out of plane modes is favoured in para positions with the largest distance between the two functional groups; (ii) the isomeric dependence of $\Delta I$ is in agreement with the isomeric frequency dependence of $v_{l}$ $\left(\Delta I_{\text {para }}<\Delta I_{\text {ortho }} \leq \Delta I_{\text {meta }} \Leftrightarrow v_{l, \text { para }}<v_{l, \text { ortho }} \leq v_{l, \text { meta }}\right)$. Finally, it can be noticed that the formation of the intramolecular HB between the $-\mathrm{OH}$ and the $-\mathrm{OCH}_{3}$ functional group in 2-MP does not affect the inertial defect of 2-MP. 


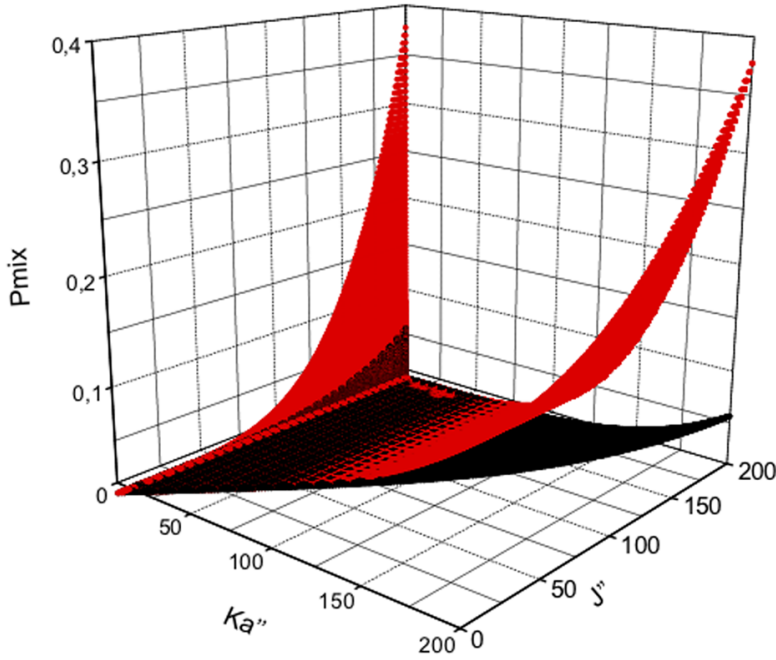

FIG. 7. 3D representation of the mixing coefficient $P_{\text {mix }}$ of 4-MP rotational energy levels of the $\mathrm{V}_{45}=2$ (red circles) and $\mathrm{V}_{44}=1$ (black circles) ES vs the $\mathrm{J}^{\prime \prime}$ and $K_{a}^{\prime \prime}$ quantum numbers. The values of $P_{\text {mix }}$ were directly obtained from the .egy file generated by the SPCAT Pickett's program. ${ }^{21}$ Projections on the $\left(\mathrm{P}_{\text {mix }} ; J^{\prime \prime}\right)$ and $\left(\mathrm{P}_{\text {mix }} ; K_{a}{ }^{\prime \prime}\right)$ planes are plotted.

\section{ES inertial defects}

In 1966, Hanyu et al. ${ }^{46}$ observed the decreasing of the inertial defect of nitrosobenzene with each excitation of the lowfrequency out of plane vibration $v_{l}$. In a first approximation, they have demonstrated that if the lowest frequency out of plane vibration has an energy sufficiently lower than the higher energy inplane modes $\left(v_{s}^{2} \gg v_{l}^{2}\right.$ see Refs. 42 and 46$)$, then the variation of the inertial defects between two adjacent out of plane modes is given by

$$
\Delta I_{v_{l}=n+1}-\Delta I_{v_{l}=n}=-\frac{h}{2 \pi^{2} c v_{l}}
$$

with $\frac{h}{2 \pi^{2} c}=67.4305 \mathrm{amu} . \AA^{2} \mathrm{~cm}^{-1}$ and $v_{l}$ the lowest energy out of plane mode wavenumber in $\mathrm{cm}^{-1}$.
TABLE V. Variation of the inertial defects $\Delta I_{\mathrm{V}_{45}=2}-\Delta I_{\mathrm{V}_{45}=1}$ in amu. $\AA^{2}$ units with the excitation of the lowest energy out of plane vibrational mode $v_{45}$. Comparison of the vibrational wavenumbers $v_{45}=1$ in $\mathrm{cm}^{-1}$ unit deduced from Eq. (2) $\mathrm{V}_{45, \Delta I}=1,42,46$ the experimental value $\mathrm{V}_{45 \text {, exp. }}=1$ obtained in Ref. 29 by FT-Far-IR spectroscopy and anharmonic calculations $\mathrm{v}_{45 \text {, calc. }}=1$ at the B3LYP/cc-pVTZ level of theory.

\begin{tabular}{lcccc}
\hline \hline Molecule & $\Delta I_{\mathrm{v}_{45}=2}-\Delta I_{\mathrm{v}_{45}=1}$ & $\mathrm{~V} 45, \Delta I=1^{42,46}$ & $\mathrm{v}_{45, \exp .}=1^{29}$ & $\mathrm{v}_{45, \text { calc. }}=1$ \\
\hline 2-MP C1 & -0.91839 & 73.4 & 63.5 & 76.2 \\
3-MP C2 & -0.87687 & 76.9 & 78.3 & 80.2 \\
4-MP C1 & -1.00308 & 67.2 & $\ldots$ & 66.5 \\
\hline \hline
\end{tabular}

As shown in Table III, the rotational constants of $\mathrm{v}_{45}=2$ and $\mathrm{v}_{44}=1 \mathrm{ES}$ (especially for 2-MP) are very close. That is not the case for their inertia defects with a value of $\Delta I_{\mathrm{V}_{45}=2}$ significantly lower than the $\Delta I_{\mathrm{v}_{44}=1}$ value according to Eq. (2), which ensures the validity of our assignment. In the other hand, it was possible to determine from the rotational constants the vibrational wavenumbers of $\mathrm{v}_{45}=1$ for the three MP isomers by using Eq. (2) of Hanyu et al. ${ }^{46}$ The results are presented in Table $V$ where the variation of the inertial defects $\Delta I_{\mathrm{V}_{45}=2}-\Delta I_{\mathrm{v}_{45}=1}$ in amu. $\AA^{2}$ units with the excitation of the lowest energy out of plane vibrational mode $v_{45}$ has been calculated for 2-MP C1, 3-MP C2, ${ }^{9}$ and 4-MP C1. Taking into account the good precision of the ES $\Delta I$ measured by mm-wave spectroscopy, this variation is determined with a high degree of accuracy and the isomeric order discussed in Sec. III C 1 is again verified $\left(\Delta I_{\mathrm{V}_{45}=2}\right.$ $\left.-\Delta I_{\mathrm{V}_{45}=1}\right)_{\text {para }}<\left(\Delta I_{\mathrm{V}_{45}=2}-\Delta I_{\mathrm{V}_{45}=1}\right)_{\text {ortho }}<\left(\Delta I_{\mathrm{V}_{45}=2}-\Delta I_{\mathrm{V}_{45}=1}\right)_{\text {meta }}$. Using Eq. (2), it was possible to determine, from negative inertial defects, the lowest energy out of plane mode wavenumber noted $\mathrm{v}_{45, \Delta I}$ in Table $\mathrm{V}$ and to compare the obtained values with experimental FT-Far-IR ${ }^{29}$ and calculated B3LYP/cc-pVTZ vibrational wavenumbers. For 2-MP C1 and 3-MP C2, the agreement of $\mathrm{v}_{45, \Delta I}$ values and the experimental measurements is better than $10 \mathrm{~cm}^{-1}$. In the case of 4-MP, the variation of the inertial defects with the excitation of the $v_{45}$ mode allows us to estimate the position of the non-observed vibrational band center at $67.2 \mathrm{~cm}^{-1}$ in very good agreement with the anharmonic calculated value of $66.5 \mathrm{~cm}^{-1}$.

TABLE IV. GS inertial defects in amu. $\AA^{2}$ units of ortho, meta, and para isomers of phenol and anisole derivatives measured by rotationally resolved high resolution spectroscopy. Only the most stable observed rotamers have been considered.

\begin{tabular}{lccccc}
\hline \hline Molecule & Functional groups & $\Delta I_{\text {ortho }}$ & $\Delta I_{\text {meta }}$ & $\Delta I_{\text {para }}$ & References \\
\hline Methoxyphenol & $\mathrm{OH} / \mathrm{OCH}_{3}$ & -3.459 & -3.428 & -3.555 & This work (ortho, para) + Ref. 9 (meta) \\
\hline $\begin{array}{l}\text { Phenol } \\
\text { Fluorophenol }\end{array}$ & $\mathrm{OH}$ & & -0.031 & & $\begin{array}{c}\text { Reference 30 } \\
\text { Dihydroxybenzene }\end{array}$ \\
$\begin{array}{l}\text { Cresol } \\
\mathrm{OH} / \mathrm{OH}\end{array}$ & -0.002 & -0.017 & -0.073 & $\begin{array}{c}\text { Reference 31 (ortho, meta) + Ref. 32 (para) } \\
\text { Reference 33 (ortho) + Ref. 34 (meta) + Ref. 35 (para) } \\
\text { Anisole }\end{array}$ \\
$\begin{array}{l}\text { Methylanisole } \\
\text { Dimethoxybenzene }\end{array}$ & $\mathrm{OCH}_{3}$ & -3.230 & -3.156 & -3.253 & Reference 36 (ortho) + Ref. 37 (meta) + Ref. 38 (para) \\
\hline \hline
\end{tabular}




\section{CONCLUSIONS}

Millimetre-wave absorption and emission rotational spectra of guaiacol (2-MP) and mequinol (4-MP) have been measured with a frequency multiplication chain and a mm-wave CPFT spectrometer, respectively. The experimental measurements were supplemented by anharmonic B3LYP/cc-pVTZ calculations. For 2-MP, only one rotamer may be observed at room temperature in the $\mathrm{mm}$-wave spectra. This conformation is strongly stabilised by the formation of an intramolecular $\mathrm{O}-\mathrm{H} \cdots \mathrm{OCH}_{3} \mathrm{HB}$ characterised in this work by a topology analysis of the electron density. The full set of GS molecular parameters including quartic corrections has been fitted allowing to reproduce $a$-type and $b$-type rotational transitions at the experimental accuracy. In a global fit including more than 30000 lines, pure rotational transitions in three ES have been assigned and the corresponding molecular constants fitted. These ES correspond to the fundamental and the overtone of the $v_{45}$ out of plane $\mathrm{O}-\mathrm{CH}_{3}$ bending and the fundamental $v_{44} \mathrm{CH}_{3}$ torsion. For 4-MP, two stable rotamers, one in configuration cis and the other one in configuration trans were expected. Due to the weak polarity of the trans structure, only the cis-rotamer was observed in the $\mathrm{mm}$-wave spectra. As for 2-MP, both GS and the three previously mentioned ES were assigned and all the molecular parameters up to quartic corrections were determined. Nevertheless, for $\mathrm{v}_{45}=2$ and $\mathrm{v}_{44}=1 \mathrm{ES}$, an interaction between the two ES was highlighted at high $J^{\prime \prime}$ and $K_{a}{ }^{\prime \prime}$ values. We have additionally discussed the negative inertial defects of the three MP isomers. A comparison of different phenol and anisole derivatives highlights an isomeric dependence of the GS $\Delta I$ according to the lowest vibrational energies of the out of plane modes. The measured $\Delta I$ allowed to avoid any ambiguity in the low-frequency vibrational ES assignment. The variation of the inertial defects with the excitation of the methoxy out of plane mode allows us to estimate with a good degree of accuracy its vibrational energy. All these results demonstrate that the negative inertial defect of aromatic compounds, measured with a sufficient precision, is a powerful tool to discriminate isomers and to assign unambiguously the rotational structures in the low-energy out of plane vibrational states. An improvement of the sensitivity of the spectrometers should allow in the near future to study the weakly volatile syringol, the other primary block of lignin released in quantity in the atmosphere during biomass fires. ${ }^{47}$ Finally, microwave, $\mathrm{THz}$ and IR spectrometers coupled to free jet supersonic beams suggest the possibility to study hydrated MP complexes as it was performed with the benzoic acid-water complex in order to bridge the gap between gas-phase and aerosols in the atmosphere towards the study of micro-solvation processes. ${ }^{48}$

\section{SUPPLEMENTARY MATERIAL}

The line lists of the assigned $\mathrm{mm}$-wave rotational transitions frequencies of 2-MP and 4-MP in the GS and ES are, respectively, presented in Tables S1 and S2 of the supplementary material.

\section{ACKNOWLEDGMENTS}

This work and the postdoc of A. Jabri took place in the Labex CaPPA (Chemical and Physical Properties of the Atmosphere) funded by the French National Research Agency (ANR) throught the PIA (Programme d'Investissements d'Avenir) under Contract
No. ANR-11-LABX-005-01. The Ph.D. of A. Roucou was supported by the Région Hauts-de-France and the French Direction Générale de l'Armement (DGA). The authors are also grateful for the financial grant received from the Région Hauts-de-France, the Ministère de l'Enseignement Supérieur et de la Recherche (CPER Climibio), and the European Fund for Regional Economic Development. ANR is also thanked for their financial support for the project Original Sub-millimetre Chirped pulse instrumentation for Astrochemical Reactivity (OSCAR) under Contract No. ANR-15-CE29-0017. The last acknowledgements go to two computational clusters: the Centre de Ressources Informatiques de Lille 1 (CRI) and CALCULCO, the computing platform from the Université du Littoral Côte d'Opale where the GAUSSIAN16 and MOLPRO calculations were performed, respectively.

\section{REFERENCES}

${ }^{1}$ C. D. Simpson, M. Paulsen, R. L. Dills, L.-J. S. Liu, and D. A. Kalman, Environ. Sci. Technol. 39, 631-637 (2005).

${ }^{2}$ A. Lauraguais, C. Coeur-Tourneur, A. Cassez, K. Deboudt, M. Fourmentin, and M. Choël, Atmos. Environ. 86, 155-163 (2014).

${ }^{3}$ W. Ahmad, C. Coeur, A. Tomas, T. Fagniez, J.-B. Brubach, and A. Cuisset, Appl. Opt. 56, E116-E122 (2017).

${ }^{4}$ S. Islam, A. Ganesan, R. Auchettl, O. Plekan, R. G. Acres, F. Wang, and K. C. Prince, J. Chem. Phys. 149, 134312 (2018).

${ }^{\mathbf{5}}$ W. Caminati, S. Melandri, and L. B. Favero, J. Mol. Spectrosc. 161, 427-434 (1993).

${ }^{6}$ J. A. Ruiz-Santoyo, M. Rodríguez-Matus, J. L. Cabellos, J. T. Yi, D. W. Pratt, M. Schmitt, G. Merino, and L. Álvarez-Valtierra, J. Chem. Phys. 143, 094301 (2015).

${ }^{7}$ S. Ullrich, W. D. Geppert, C. E. Dessent, and K. Müller-Dethlefs, J. Phys. Chem. A 104, 11864-11869 (2000).

${ }^{8}$ M. Wilke, M. Schneider, J. Wilke, J. A. Ruiz-Santoyo, J. J. Campos-Amador, M. E. González-Medina, L. Álvarez-Valtierra, and M. Schmitt, J. Mol. Struct. 1140, 59-66 (2017).

${ }^{9}$ A. Roucou, D. Fontanari, G. Dhont, A. Jabri, C. Bray, F. Hindle, G. Mouret, R. Bocquet, and A. Cuisset, ChemPhysChem 19, 1572-1578 (2018).

${ }^{10}$ S. Kim, P. A. Thiessen, E. E. Bolton, J. Chen, G. Fu, A. Gindulyte, L. Han, J. He, S. He, B. A. Shoemaker et al., Nucleic Acids Res. 44, D1202-D1213 (2015).

${ }^{11}$ G. Mouret, M. Guinet, A. Cuisset, L. Croize, S. Eliet, R. Bocquet, and F. Hindle, IEEE Sens. J. 13, 133-138 (2013).

${ }^{12}$ F. Hindle, C. Bray, K. Hickson, D. Fontanari, M. Mouelhi, A. Cuisset, G. Mouret, and R. Bocquet, J. Infrared, Millimeter, Terahertz Waves 39, 105-119 (2018).

${ }^{13}$ A. M. Daly, L. Kolesnikova, S. Mata, and J. L. Alonso, J. Mol. Spectrosc. 306, 11-18 (2014).

${ }^{14}$ M. Martin-Drumel, F. Hindle, G. Mouret, A. Cuisset, and J. Cernicharo, Astrophys. J. 799, 115 (2015).

${ }^{15}$ J. L. Neill, B. J. Harris, A. L. Steber, K. O. Douglass, D. F. Plusquellic, and B. H. Pate, Opt. Express 21, 19743-19749 (2013).

${ }^{16}$ M. Frisch, G. Trucks, H. Schlegel, G. Scuseria, M. Robb, J. Cheeseman, G. Scalmani, V. Barone, G. Petersson, H. Nakatsuji et al., Gaussian 16, Revision a. 03, Gaussian, Inc., Wallingford, CT, 2016.

${ }^{17}$ R. A. Kendall, T. H. Dunning, Jr., and R. J. Harrison, J. Chem. Phys. 96, 6796-6806 (1992).

${ }^{18}$ L. Cesari, L. Canabady-Rochelle, and F. Mutelet, Struct. Chem. 29, 179-194 (2018).

${ }^{19}$ H.-J. Werner, P. J. Knowles, G. Knizia, F. R. Manby, and M. Schütz, Wiley Interdiscip. Rev.: Comput. Mol. Sci. 2, 242-253 (2012).

${ }^{20}$ T. Lu and F. Chen, J. Comput. Chem. 33, 580-592 (2012).

${ }^{21}$ H. M. Pickett, J. Mol. Spectrosc. 148, 371-377 (1991).

${ }^{22}$ Z. Kisiel, E. Białkowska-Jaworska, and L. Pszczółkowski, J. Chem. Phys. 109, 10263-10272 (1998). 
${ }^{23}$ Z. Kisiel, E. Białkowska-Jaworska, and L. Pszczółkowski, J. Mol. Spectrosc. 199, 5-12 (2000).

${ }^{24}$ L. Ferres, H. Mouhib, W. Stahl, and H. V. L. Nguyen, ChemPhysChem 18, 1855-1859 (2017).

${ }^{25}$ L. Ferres, W. Stahl, and H. V. L. Nguyen, J. Chem. Phys. 148, 124304 (2018).

${ }^{26}$ L. Ferres, W. Stahl, I. Kleiner, and H. V. L. Nguyen, J. Mol. Spectrosc. 343, 44-49 (2018).

${ }^{27}$ A. Cuisset, M.-A. M. Drumel, F. Hindle, G. Mouret, and D. A. Sadovskií, Chem. Phys. Lett. 586, 10-15 (2013).

${ }^{28}$ A. Roucou, G. Dhont, A. Cuisset, M.-A. Martin-Drumel, S. Thorwirth, D. Fontanari, and W. L. Meerts, J. Chem. Phys. 147, 054303 (2017).

${ }^{29}$ A. Cuisset, C. Coeur, G. Mouret, W. Ahmad, A. Tomas, and O. Pirali, J. Quant. Spectrosc. Radiat. Transfer 179, 51-58 (2016).

${ }^{30}$ C. Tanjaroon and S. G. Kukolich, J. Phys. Chem. A 113, 9185-9192 (2009).

${ }^{31}$ A. Bell, J. Singer, D. Desmond, O. Mahassneh, and J. van Wijngaarden, J. Mol. Spectrosc. 331, 53-59 (2017).

${ }^{32}$ C. Ratzer, M. Nispel, and M. Schmitt, Phys. Chem. Chem. Phys. 5, 812-819 (2003).

${ }^{33}$ W. Caminati, S. Di Bernardo, L. Schäfer, S. Q. Kulp-Newton, and K. Siam, J. Mol. Struct. 240, 263-274 (1990).

${ }^{34}$ S. Melandri, G. Maccaferri, W. Caminati, and P. G. Favero, Chem. Phys. Lett. 256, 513-517 (1996).
${ }^{35}$ W. Caminati, S. Melandri, and L. B. Favero, J. Chem. Phys. 100, 8569-8572 (1994).

${ }^{36}$ A. Welzel, A. Hellweg, I. Merke, and W. Stahl, J. Mol. Spectrosc. 215, 58-65 (2002).

${ }^{37}$ A. Hellweg, C. Hättig, I. Merke, and W. Stahl, J. Chem. Phys. 124, 204305 (2006).

${ }^{38}$ A. Hellweg and C. Hättig, J. Chem. Phys. 127, 024307 (2007).

${ }^{39}$ B. Reinhold, I. Finneran, and S. Shipman, J. Mol. Spectrosc. 270, 89-97 (2011).

${ }^{40}$ M. Schneider, M. Wilke, M.-L. Hebestreit, C. Henrichs, W. L. Meerts, and M. Schmitt, ChemPhysChem 19, 307-318 (2018).

${ }^{41}$ O. Pirali, Z. Kisiel, M. Goubet, S. Gruet, M. Martin-Drumel, A. Cuisset, F. Hindle, and G. Mouret, J. Chem. Phys. 142, 104310 (2015).

${ }^{42}$ T. Oka, J. Mol. Struct. 352, 225-233 (1995).

${ }^{43}$ T. Oka and Y. Morino, J. Mol. Spectrosc. 6, 472-482 (1961).

${ }^{44}$ S. Gruet, M. Goubet, and O. Pirali, J. Chem. Phys. 140, 234308 (2014).

${ }^{45}$ M. K. Jahn, J.-U. Grabow, M. J. Travers, D. Wachsmuth, P. D. Godfrey, and D. McNaughton, Phys. Chem. Chem. Phys. 19, 8970-8976 (2017).

${ }^{46}$ Y. Hanyu, C. O. Britt, and J. E. Boggs, J. Chem. Phys. 45, 4725-4728 (1966).

${ }^{47}$ J. D. Young, M. Staniforth, J. C. Dean, G. M. Roberts, F. Mazzoni, T. N. Karsili, M. N. Ashfold, T. S. Zwier, and V. G. Stavros, J. Phys. Chem. Lett. 5, 2138-2143 (2014).

${ }^{48}$ E. G. Schnitzler and W. Jäger, Phys. Chem. Chem. Phys. 16, 2305-2314 (2014).

${ }^{49}$ A. Roucou, I. Kleiner, M. Goubet, S. Bteich, G. Mouret, R. Bocquet, F. Hindle, W. L. Meerts, and A. Cuisset, ChemPhysChem 19, 1056-1067 (2018). 